\title{
CTA for Screening of Complicated Atherosclerotic Carotid Plaque-American Heart Association Type VI Lesions as Defined by MRI
}

M. Trelles, K.M. Eberhardt, M. Buchholz, A. Schindler, A. Bayer-Karpinska, M. Dichgans, M.F. Reiser, K. Nikolaou, and T. Saam

\begin{abstract}
BACKGROUND AND PURPOSE: High-resolution carotid MR imaging can accurately identify complicated American Heart Association lesion type VI plaques, which are characterized by thrombus, hemorrhage, or a ruptured fibrous cap. The purpose of this study is to evaluate whether CTA can be used as screening tool to predict the presence or absence of American Heart Association lesion type VI plaques as defined by high-resolution MR imaging.
\end{abstract}

METHODS: Fifty-one patients with suspected ischemic stroke or TIA with carotid CTA and carotid MR imaging performed within 14 days of the event/admission from April 2008 to December 2010 were reviewed. Vessels with stents or occlusion were excluded ( $n=2)$. Each carotid artery was assigned an American Heart Association lesion type classification by MR imaging. The maximum wall thickness, maximum soft plaque component thickness, maximum calcified component thickness, and its attenuation (if the soft plaque component thickness was $>2 \mathrm{~mm}$ ) were obtained from the CTA.

RESULTS: The maximum soft plaque component thickness proved the best discriminating factor to predict a complicated plaque by MR imaging, with a receiver operating characteristic area under the curve of 0.89 . The optimal sensitivity and specificity for detection of complicated plaque by MR imaging was achieved with a soft plaque component thickness threshold of $4.4 \mathrm{~mm}$ (sensitivity, 0.65 ; specificity, 0.94; positive predictive value, 0.75 ; and negative predictive value, 0.9$)$. No complicated plaque had a soft tissue plaque thickness $<2.2 \mathrm{~mm}$ (negative predictive value, 1) and no simple (noncomplicated) plaque had a thickness $>5.6 \mathrm{~mm}$ (positive predictive value, 1).

CONCLUSIONS: Maximum soft plaque component thickness as measured by carotid CTA is a reliable indicator of a complicated plaque, with a threshold of $2.2 \mathrm{~mm}$ representing little to no probability of a complicated American Heart Association lesion type VI plaque.

ABBREVIATIONS: AHA-LT6 $=$ American Heart Association type VI; IPH $=$ intraplaque hemorrhage; $\mathrm{HU}=$ Hounsfield units

M acroangiopathic disease causes $20-30 \%$ of ischemic strokes, most occurring as the result of plaque rupture. ${ }^{1,2}$ This percentage might even be higher, as a recent MR imaging study ${ }^{3}$ suggested that a substantial portion of patients with cryptogenic strokes have ipsilateral complicated American Heart Association type VI (AHA-LT6) carotid plaques, characterized by intraplaque hemorrhage (IPH), thrombus, or a ruptured fibrous cap. These characteristics are currently identifiable on high-reso-

Received December 6, 2012; accepted after revision March 20, 2013.

From the Department of Radiology (M.T.), University of Texas Medical Branch, Galveston, Texas; and Institute for Clinical Radiology (K.M.E., M.B., A.S., M.F.R., K.N., T.S.) and Institute for Stroke and Dementia Research (A.B.-K., M.D.), Ludwig-Maximilians-University, Munich, Germany.

Please address correspondence to Tobias Saam, MD, Institute for Clinical Radiology, Ludwig-Maximilians-University Hospitals Munich, Pettenkoferstr. 8a, 80336 München, Germany; e-mail: mitrelle@utmb.edu

三 Indicates article with supplemental on-line table

Indicates article with supplemental on-line figure

http://dx.doi.org/10.3174/ajnr.A3607 lution MR imaging of the carotid arteries ${ }^{4,5}$ with improved sensitivity and specificity with the use of specialized high-resolution techniques. ${ }^{4,6-9}$ Furthermore, several MR studies have demonstrated that AHA-LT6 plaques are related to increased risk of stroke independent of the severity of luminal stenosis. ${ }^{10,11}$ Highresolution MR imaging of the carotid bifurcation, however, is time-consuming, requires specialized coils, and is not widely available.

Carotid sonography is often used as a first-line imaging technique to screen for the presence or absence of atherosclerosis, but this method is highly operator-dependent and provides limited information regarding plaque morphology and complications. ${ }^{12-14}$ Operators usually focus on the degree of stenosis, and intima-media thickness measurements, plaque thickness, and morphology are not considered part of the routine sonography evaluation.

CT of the brain is commonly used as part of the routine clinical work-up in patients with suspected ischemic stroke or TIA. CTA 
of the circle of Willis and cervical carotid arteries is frequently performed concomitantly to evaluate for thrombus and to define the degree of carotid stenosis, ${ }^{15}$ which is the only broadly accepted finding used to indicate endarterectomy. ${ }^{16-18}$ Furthermore, carotid CTA provides high-resolution images of the carotid bulb to identify ulcerations ${ }^{19-21}$ and it also provides information about aortic arch plaques, which have been associated with thromboembolic events. $^{22}$

MDCT angiography is also able to differentiate between soft, mixed, and hard plaques, with questionable evaluation of fibrous tissue and lipid-rich necrotic core. ${ }^{23-25}$ The use of Hounsfield units (HU) analysis for the identification of the components is limited secondary to overlap in $\mathrm{HU}$ values between different plaque components and the presence of artifacts from calcified plaque and the enhanced arterial lumen. ${ }^{26,27}$ To date, CTA is not able to reliably identify complicated AHA-TL6 plaques, which are characterized by fibrous cap rupture, attached thrombus, or hemorrhage, and are the imaging findings most closely related with an increased clinical risk of stroke. ${ }^{11}$ Thus, it would be beneficial to evaluate imaging characteristics found on CTA that would warrant obtaining a high-resolution MR imaging of the carotid bifurcation.

The purpose of this study is to evaluate whether carotid CTA can be used as a screening tool to predict the presence or absence of a complicated AHA-LT6 carotid bulb plaque with fibrous cap rupture, attached thrombus, or hemorrhage as defined by highresolution MR imaging.

\section{MATERIALS AND METHODS Patient Selection}

Patients with suspected ischemic stroke or TIA of the anterior circulation who had a carotid CTA and high-resolution carotid MR imaging performed within 14 days of the event or admission from April 2008 to December 2010 were included. Patients with imaging studies of insufficient quality and vessels with prior endarterectomy, stent, or occlusion were excluded. The study was approved by the local ethics committee, and all subjects gave written informed consent. CT brain imaging was routinely performed immediately after presentation to assess intracerebral hemorrhage and acute ischemic stroke.

\section{Imaging Protocols and Analysis}

Because assessment of patients with stroke is performed by several units in our clinical center, scans were performed on various CT scanners: Bright Speed S (GE Healthcare, Milwaukee, Wiscon$\sin$ ), Aquilion (Toshiba Medical Systems, Tokyo, Japan), Somatom Definition Flash (Siemens, Erlangen, Germany), Somatom Definitition AS+ (Siemens), and Sensation 64 (Siemens). All CTA images were obtained with coverage at least from the aortic arch to the cranium by use of the respective standard protocol parameters. Nonionic iodinated contrast material was applied intravenously, adjusted to patient weight (Ultravist 370; BayerSchering Pharma, Berlin, Germany, ie, 0.35-0.50 g iodine per $\mathrm{kg}$ body weight at an injection rate of $4.5-6 \mathrm{~mL} / \mathrm{s}$ followed by $100 \mathrm{~mL}$ of saline at identical flow) (On-line Table).

Participants underwent high-resolution black-blood carotid MR imaging within a mean of 7.8 days after the CT scan. All subjects were imaged by use of a previously published multisequence protocol ${ }^{24}$ (TOF MR angiography, axial precontrast and postcontrast black-blood T1, proton attenuation, and T2WI sequences with fat saturation centered at the carotid bifurcation; best in-plane resolution, $0.5 \times 0.5 \mathrm{~mm}^{2}$ ) at $3 \mathrm{~T}$ (Magnetom Verio, Siemens). To improve signal-to-noise performance and optimize spatial resolution, a dedicated 4-channel surface coil (Machnet B.V., Eelde, The Netherlands) was used for bilateral carotid scans. Gadolinium-DO3A-Butrol (Gadobutrol, Bayer Schering) of 0.1 $\mathrm{mmol} / \mathrm{kg}(0.1 \mathrm{~mL} / \mathrm{kg})$ was given at a rate of $3 \mathrm{~mL} / \mathrm{s}$.

An experienced radiologist blinded to the MR imaging results (M.T.) reviewed the axial sections of the CTAs to obtain the following measurements: maximum plaque wall thickness, maximum thickness of soft plaque component, and maximum thickness of calcified plaque component (On-line Figure). If the soft plaque component thickness was $>2 \mathrm{~mm}, 2$ oval ROIs were obtained from the plaque area measuring the mean, minimum, and maximum $\mathrm{HU}$ and the standard deviation. Window level settings were set at $800 / 200$, though small adjustments were allowed. The ROI size was kept $\geq 2 \mathrm{~mm}^{2}$. Superimposition of both ROIs was allowed in cases with small plaques. The ROIs were taken with care to include the area showing less attenuation, avoiding calcifications and the enhanced lumen. The presence of ulceration or thrombus was also noted. Ulcerations were defined as an outpouching of contrast material $>1 \mathrm{~mm} .{ }^{28}$ For completeness, the percentage of stenosis obtained by the NASCET method was also calculated.

To assess for the reproducibility of the axial measurements, a second experienced radiologist (K.M.E.) who was blinded to the initial results repeated the measurements in the original axial sections in 20 patients ( 40 carotid arteries). Furthermore, to explore the potential of measurements in a plane orthogonal to the lumen and to exclude a systematic bias of the axial measurements, curved planar reformatted images of the same 20 patients were uploaded in a separate session on a dedicated workstation (IMPAX Volume Viewing 2.2; Agfa, Greenville, South Carolina) and all plaque thickness measurements were repeated in an orthogonal plane. An image-quality rating (4-point scale, 1 = nondiagnostic, 2 = moderate, $3=$ good, $4=$ excellent) was assigned to all MR images. The MR imaging data were classified according to the modified American Heart Association lesion type classification as previously defined by Cai et $\mathrm{al}^{29}$ by 2 experienced radiologists (T.S. and K.N.) who were blinded to the CTA data. In the case of discrepancy, a consensus decision was made. Patients with image quality $\geq 2$ by MR imaging were included. The presence of complications such as hemorrhage, fibrous cap rupture, ulceration, calcific nodule, or thrombus was also annotated.

\section{Statistical Analysis}

Categoric variables are presented as percentages; continuous variables are presented as mean $\pm 95 \%$ confidence interval. Data analysis was performed by use of SPSS Statistics (Version 20; IBM, Armonk, New York). Graphs were constructing with the use of Excel (Version 14; Microsoft, Redmond, Washington). Continuous data were compared with the unpaired Student $t$ test. Categoric data were compared by means of $\chi^{2}$ test. Inter-reader and intermeasurement agreement (between measurements in the ax- 
Table 1: Patient characteristics

\begin{tabular}{lc}
\hline & $\begin{array}{c}\text { Study Population } \\
(\boldsymbol{n}=\mathbf{5 1})\end{array}$ \\
\hline Demographic & \\
Age, $y$ & $71.3 \pm 0.9$ \\
Male sex & $74 \%$ \\
$\quad$ Body mass index & $26.0 \pm 0.3$ \\
CT imaging & \\
$\quad$ Stenosis, \% (NASCET) & $26.7 \% \pm 5.6 \%$ \\
Cardiovascular risk factors & \\
Smoker & $25.5 \%(13)$ \\
Ex-smoker & $41.2 \%(21)$ \\
Hypertension & $76.5 \%(39)$ \\
Hypercholesterolemia & $45.1 \%(23)$ \\
Diabetes & $23.5 \%(12)$ \\
Coronary heart disease & $13.7 \%(7)$ \\
Family history of cardiovascular events & $31.4 \%(16)$ \\
\hline
\end{tabular}

Note:-Values are mean $+\mathrm{Cl}$ unless otherwise shown ial plane and a plane orthogonal to the vessel lumen) was determined by the intraclass correlation coefficient. A value of $P<.05$ was considered statistically significant. Receiver operating characteristic curves were constructed to evaluate the ability of the different imaging characteristics to predict the presence of complicated plaque by MR imaging. The optimal sensitivity and specificity were calculated together with the area under the curve.

\section{RESULTS}

\section{Patients}

Fifty-four patients were included (mean age of 71 years, male/ female ratio of 2.9/1). Three MR imaging scans (with 6 carotid vessels in total) were excluded because of insufficient image quality. One carotid vessel with a stent and 1 carotid vessel with total

Table 2: General plaque characteristics by MRI-derived AHA lesion type

\begin{tabular}{|c|c|c|c|c|c|c|c|}
\hline $\begin{array}{c}\text { MRI-AHA } \\
\text { Lesion Type }\end{array}$ & $\begin{array}{l}\text { Maximum Wall } \\
\text { Thickness }\end{array}$ & $\begin{array}{c}\text { Maximum Soft } \\
\text { Plaque Thickness }\end{array}$ & $\begin{array}{l}\text { Maximum Hard } \\
\text { Plaque Thickness }\end{array}$ & $\begin{array}{l}\text { Minimum } \\
\text { Density }\end{array}$ & $\begin{array}{l}\text { Maximum } \\
\text { Density }\end{array}$ & $\begin{array}{c}\text { Mean } \\
\text { Density }\end{array}$ & $\begin{array}{c}\text { NASCET Percentage } \\
\text { Stenosis }\end{array}$ \\
\hline$I-I I$ & $2.2 \pm 0.6$ & $1.8 \pm 0.6$ & $1.4 \pm 0.8$ & $38 \pm N A^{a}$ & $197 \pm N^{a}$ & $82 \pm N A^{a}$ & $0.0 \% \pm \mathrm{NA}^{\mathrm{a}}$ \\
\hline III & $3.7 \pm 0.5$ & $1.8 \pm 0.8$ & $3.0 \pm 0.5$ & $41 \pm 18$ & $224 \pm 68$ & $114 \pm 29$ & $15.1 \% \pm 12.5 \%$ \\
\hline IV-V & $4.0 \pm 0.3$ & $2.9 \pm 0.5$ & $2.4 \pm 0.5$ & $14 \pm 10$ & $134 \pm 21$ & $59 \pm 12$ & $21.1 \% \pm 8.0 \%$ \\
\hline VI & $5.3 \pm 0.4$ & $4.7 \pm 0.5$ & $2.5 \pm 0.5$ & $18 \pm 9$ & $143 \pm 18$ & $60 \pm 12$ & $46.3 \% \pm 10.6 \%$ \\
\hline VII & $4.4 \pm 0.7$ & $1.1 \pm 0.5$ & $3.9 \pm 0.5$ & $31 \pm 8$ & $215 \pm 33$ & $91 \pm 11$ & $33.1 \% \pm 14.0 \%$ \\
\hline Total & $4.2 \pm 0.3$ & $2.8 \pm 0.4$ & $2.7 \pm 0.3$ & $20 \pm 6$ & $152 \pm 17$ & $67 \pm 9$ & $26.7 \% \pm 5.6 \%$ \\
\hline
\end{tabular}

Note:-Thickness is given in millimeters; density of the soft plaque component in Hounsfield units (HU) $\pm \mathrm{Cl}$. AHA indicates American Heart Association.

a Only $1 \mathrm{HU}$ measurement was obtained for AHA lesion type I/II, therefore no Cls are reported.

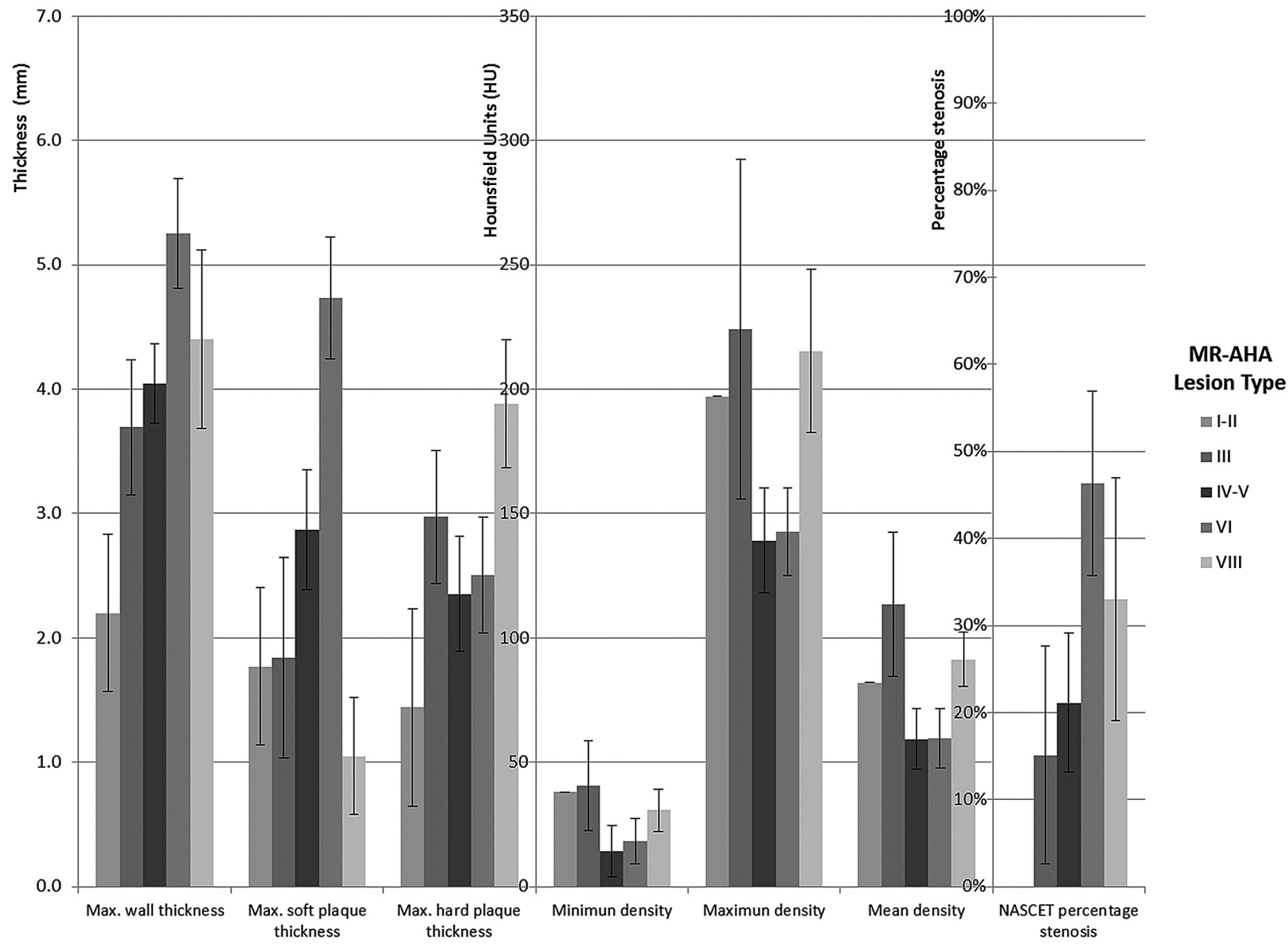

FIG 1. General plaque characteristics by MR imaging-derived American Heart Association lesion type. Thickness is given in centimeters, density in Hounsfield units, stenosis in percentage (\%) \pm confidence interval. 
occlusion were also excluded. This resulted in a total of $100 \mathrm{ca}-$ rotid vessels in 51 patients for analysis. Patient information is shown in Table 1.

\section{Carotid Plaque Lesion Type Classification}

Twenty-three carotid arteries had a complicated AHA-LT6 by MR imaging, with the presence of IPH, thrombus, or a ruptured fibrous cap. Seven arteries were classified as type I, 14 as type III, 38 as type IV/V, and 18 as type VII plaques. No plaque was classified as a type VIII plaque; 67 of 100 vessels had a maximum thickness plaque component $>2 \mathrm{~mm}$ and thus $\mathrm{HU}$ measurements were performed. All of the complicated plaques had a soft plaque thickness $>2 \mathrm{~mm}$ and thus HU was measured.

Table 3: General plaque characteristics of noncomplicated versus complicated AHA-LT6 by MRI

\begin{tabular}{lccc}
\hline & AHA-LT6 & Others & $\boldsymbol{P}$ Value \\
\hline Maximum wall thickness & $5.3 \pm 0.4$ & $3.9 \pm 0.3$ & $(<.001)$ \\
Maximum soft plaque thickness & $4.7 \pm 0.5$ & $2.2 \pm 0.3$ & $(<.001)$ \\
Maximum hard plaque thickness & $2.5 \pm 0.5$ & $2.7 \pm 0.3$ & $(.24)$ \\
Minimum density & $18 \pm 9$ & $21 \pm 9$ & $(.36)$ \\
Maximum density & $143 \pm 18$ & $162 \pm 24$ & $(.16)$ \\
Mean density & $60 \pm 12$ & $72 \pm 12$ & $(.12)$ \\
NASCET percentage stenosis & $46.3 \% \pm 10.6 \% 20.5 \% \pm 6.0 \%$ & $(<.001)$ \\
Presence of ulcer & $15.8 \% \pm 19.5 \% 34.8 \% \pm 7.8 \%$ & $(.02)$ \\
\hline
\end{tabular}

Note:-Thickness is given in millimeters; density in Hounsfield Units $\pm \mathrm{Cl}$.
Table 2 and Fig 1 show the CTA-based plaque characteristics average results for the maximum plaque wall thickness, maximum thickness of soft plaque component, and maximum thickness of calcified plaque component as well as the average HU for the mean, maximum, and minimum densities and the average NASCET percentage stenosis according to the MR imagingbased assessment of the AHA lesion type. Confidence intervals are also reported. In general, maximum thickness and maximum soft plaque thickness increased from type I/II to type VI lesions and slightly decreased in type VII lesions, which are predominantly calcified. Therefore, the hard plaque component was largest in type VII lesions. Densities of the plaques were lower in the plaques with a lipid-rich necrotic core by MR imaging such as type IV/V and type VI compared with the lesions that were classified as predominantly fibrous by MR imaging, such as type III lesions or the soft component of type VII lesions $(P=.02)$. The average percentage of stenosis also increased from type I/II (nonstenotic), reaching a maximum with AHA-LT6. Lesion type VII showed a slight decrease in average percentage stenosis compared with AHA-LT6.

Table 3 compares complicated (AHA LT-6) and noncomplicated plaques. The average maximum thickness of the soft plaque component, the average maximum wall thickness, the NASCET

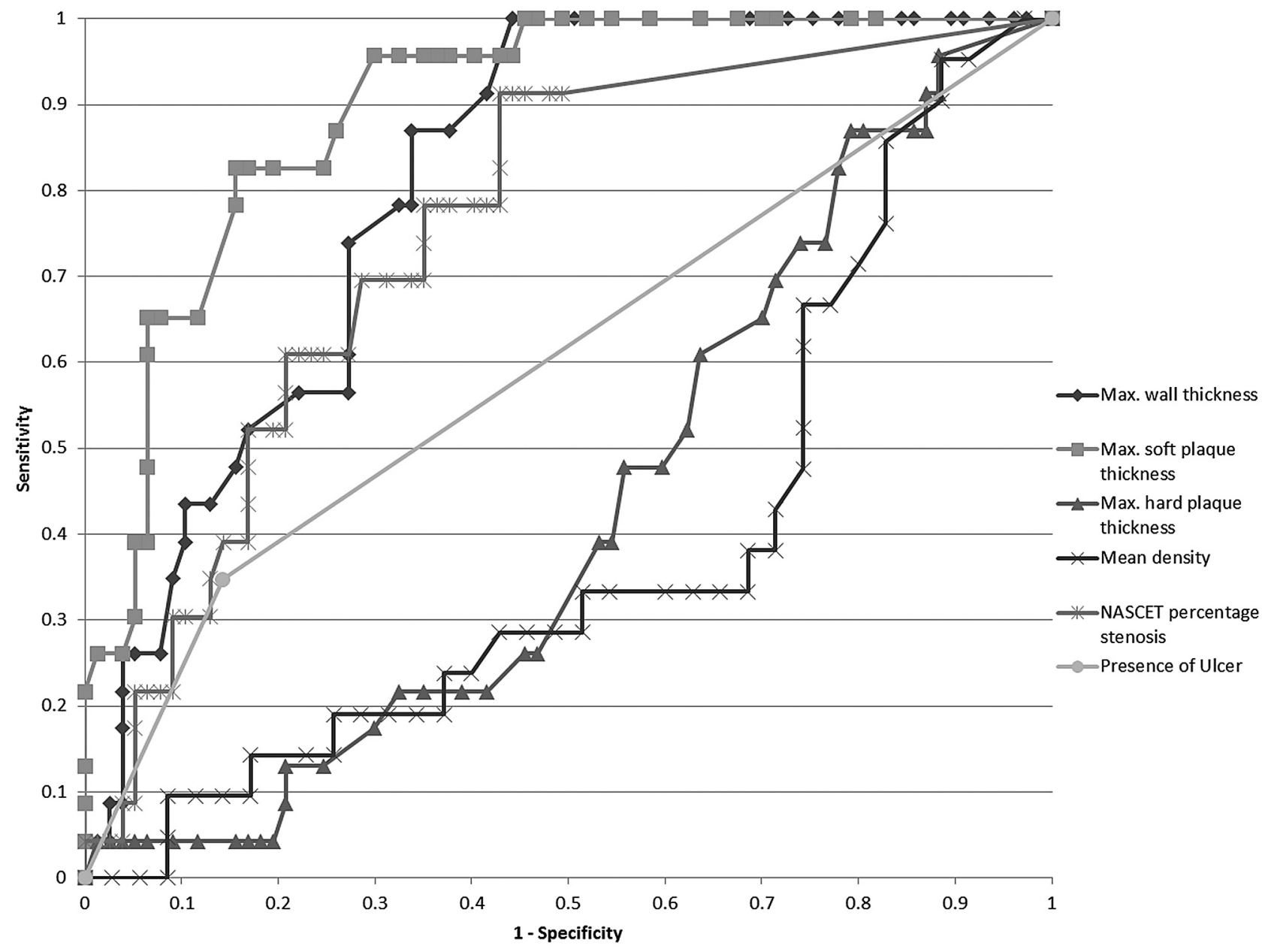

FIG 2. Receiver operating characteristic curves for the performance of the maximum wall thickness, maximum soft plaque thickness, maximum hard plaque thickness, mean attenuation NASCET percentage stenosis values, and presence of ulceration in screening for complicated AHA-LT6 plaques (area under the curve, $0.810 \pm 0.084,0.897 \pm 0.063,0.433 \pm 0.123,0.384 \pm 0.155,0.756 \pm 0.106$, and $0.602 \pm 0.139$, respectively). 

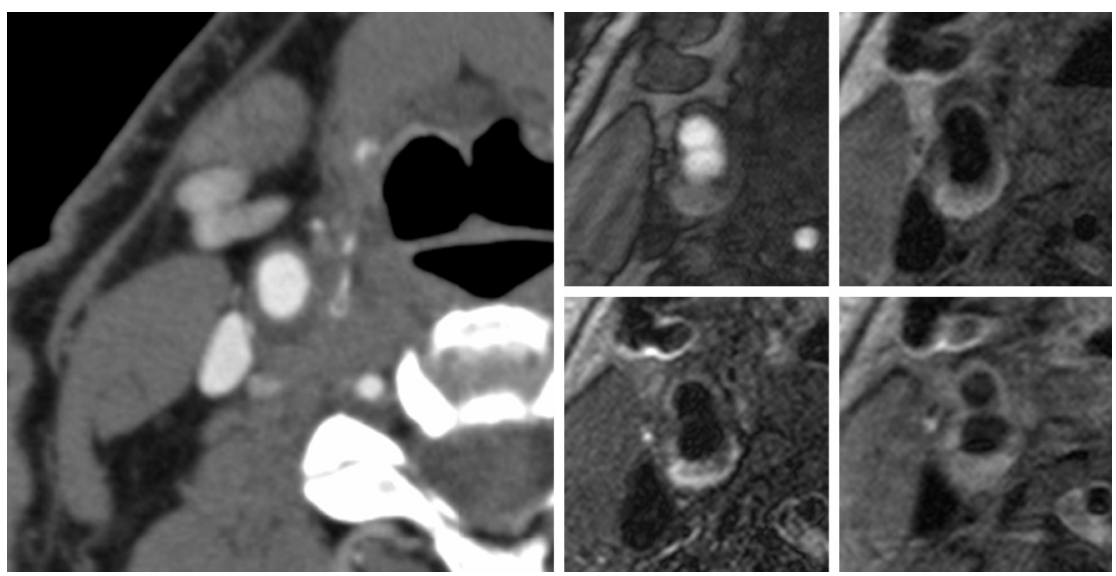

FIG 3. CTA image through a complicated AHA-LT6 plaque in the right carotid artery at the carotid bifurcation shows a soft plaque with a mean attenuation of $16 \mathrm{HU}$ (range, -7 to 46 ) and a thickness of the soft plaque component of $6 \mathrm{~mm}$. TOF, T1, T2, and T1 postgadolinium images at the same level show a hyperintense plaque on all 4 weightings, which suggests the presence of type II IPH.
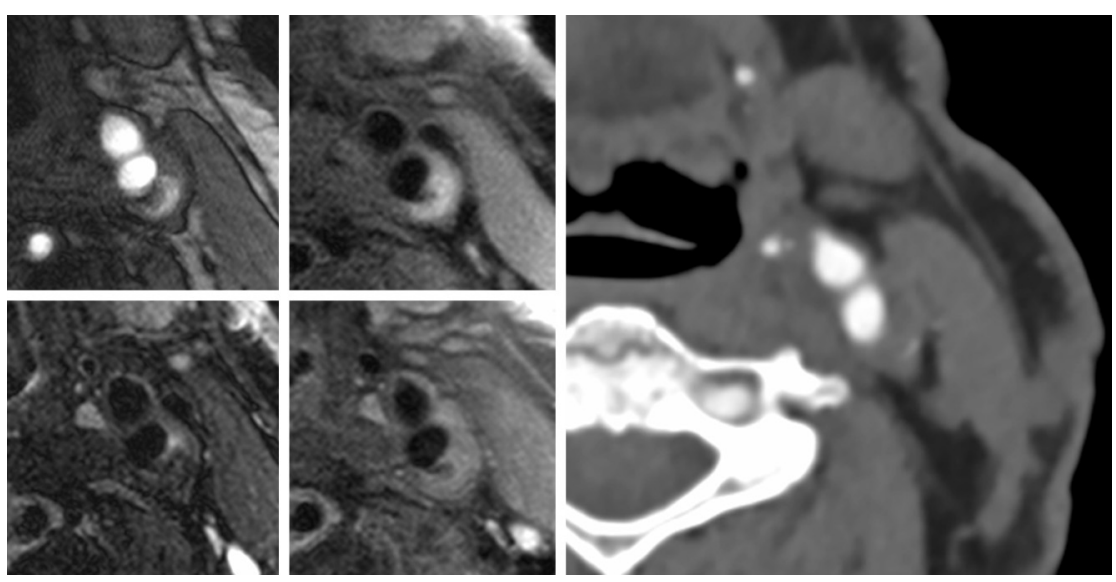

FIG 4. TOF, $T 1, T 2$, and $T 1$ postgadolinium images of a complicated AHA-LT6 plaque in the left internal carotid artery. The plaque is hyperintense on TOF and TIWI and hypointense on T2WI, which suggests the presence of type I IPH. The CT image at the identical location shows a high attenuation soft plaque with a mean attenuation of $51 \mathrm{HU}$ (range, 29-144) and a thickness of the soft plaque component of $8 \mathrm{~mm}$.

percentage stenosis, and presence of ulcerations were significantly higher in complicated plaques compared with noncomplicated plaques $(4.7 \pm 0.5$ versus $2.2 \pm 0.3 \mathrm{~mm}, P<.001$; $5.3 \pm 0.4$ versus $3.9 \pm 0.3 \mathrm{~mm}, P<.001 ; 46.3 \% \pm 10.6 \%$ versus $20.5 \% \pm 6.0 \%, P<.001$; and $15.8 \% \pm 19.5 \%$ versus $34.8 \% \pm$ $7.8 \%, P=.02$, respectively). The mean the soft plaque component attenuation did not differ significantly between complicated and noncomplicated plaques $(60 \pm 12$ versus $72 \pm 12$ HU, $P=.12)$.

The maximum soft plaque thickness was the best single discriminating factor to predict a complicated plaque by MR imaging, with a relative receiver operating characteristic area under the curve of $0.90 \pm 0.06$. Maximum wall thickness, NASCET percentage stenosis, and presence of ulceration performed less adequately with receiver operating characteristic area under the curve of $0.81 \pm 0.08,0.76 \pm 0.11$, and $0.60 \pm 0.14$, respectively. Hard plaque component thickness and HU measurements were not of diagnostic help. Utilization of maximum, minimum, and average $\mathrm{HU}$ values made no difference in the discriminatory power. Fig 2 shows these multiple receiver operating characteristic curves. Although the presence of ulceration showed a statistically significant relationship with the presence of complicated AHA-LT6 plaques, it performed poorly from a clinical standpoint (sensitivity, 0.35 ; specificity, 0.86 ; positive predictive value, 0.42 ; and negative predictive value, 0.81 ).

Figs 3 and 4 show MR and CTA images of 2 complicated AHA-LT6 plaques by MR imaging. Although CTA images show plaques with distinct densities (16 versus $54 \mathrm{HU}$ ), MR imaging identifies IPH in both lesions representing complicated AHA-LT6 plaques.

Optimal sensitivity and specificity for detection of complicated plaque by MR imaging was achieved with a soft plaque component thickness threshold of $4.4 \mathrm{~mm}$ (sensitivity, 0.65; specificity, 0.94 ; positive predictive value, 0.75 ; and negative predictive value, 0.9). Furthermore, of the 42 arteries with a soft plaque thickness $<2.2 \mathrm{~mm}$, no complicated AHA-LT6 plaque was identified by MR imaging (negative predictive value, 1 ), and of the 5 arteries with a soft plaque thickness $>5.6$ $\mathrm{mm}$ all plaques were complicated AHA-LT6 plaques (positive predictive value, 1$)$.

\section{Interreader and Intermeasurement Agreement}

The interreader intraclass correlation coefficient between reviewers 1 and 2 for axial measurements was 0.94 (0.89$0.97), 0.64(0.48-0.83)$, and $0.95(0.91-0.98)$ for the measurements of maximum plaque wall thickness, maximum thickness of calcified plaque component, and maximum thickness of soft plaque component (Table 4). When axial measurements were compared with measurements obtained at an orthogonal plane to the lumen, the intraclass correlation coefficients were 0.86 (0.75-0.92), $0.80(0.64-0.89)$, and $0.93(0.87-0.96)$ for reader 1 and $0.93(0.87-0.96), 0.69(0.47-0.82)$, and $0.97(0.94-0.98)$ for reader 2 .

\section{DISCUSSION}

The current study demonstrates that an easy-to-obtain CTA measurement, the maximum width of the soft tissue component of the atherosclerotic plaque on CTA images, is well suited to screen for the presence of complicated AHA-LT6 plaques. The optimal sensitivity and specificity for detection of complicated plaque by MR imaging was achieved with a soft plaque component thickness threshold of $4.4 \mathrm{~mm}$. More importantly, no plaque with a soft plaque component thickness $<2.2 \mathrm{~mm}$ showed signs of compli- 
Table 4: Interreader and intermeasurement intraclass correlation coefficients for maximum wall, calcified plaque, and soft plaque thickness

\begin{tabular}{lcc}
\hline & $\begin{array}{c}\text { First Axial Reading versus } \\
\text { Orthogonal Intraclass } \\
\text { Correlation Coefficient }\end{array}$ & $\begin{array}{c}\text { Second Axial Reading } \\
\text { Versus Orthogonal Intraclass } \\
\text { Correlation Coefficient }\end{array}$ \\
\hline Maximum wall thickness & $0.94(0.89-0.97)$ & $0.86(0.75-0.92)$ \\
Maximum calcified plaque thickness & $0.69(0.48-0.83)$ & $0.80(0.64-0.89)$ \\
Maximum soft plaque thickness & $0.95(0.91-0.98)$ & $0.93(0.87-0.96)$ \\
\hline
\end{tabular}

cation by MR imaging. Thus, this easy-to-obtain parameter may be used to screen for the presence of complicated AHA-LT6. Furthermore, all arteries with a soft plaque thickness $>5.6 \mathrm{~mm}$ were complicated AHA-LT6 plaques, which suggests that this plaque thickness is a good indicator for the presence of complicated atherosclerotic lesions.

Recent studies have shown a strong correlation between the maximal wall thickness and risk for new carotid stroke. ${ }^{30,31}$ This correlation could-at least in part-be explained by findings from our study, which suggest a strong correlation of all thickness measurements and presence of complicated AHA-LT6. This is also consistent with previous CTA studies that suggested a strong correlation between volumetric size of the soft plaque component and presence of ulceration ${ }^{28}$ and between presence of ulceration and IPH as described by MR imaging. ${ }^{26}$ However, we also showed that the maximum thickness of the soft plaque component performed better than the above-mentioned imaging characteristics in the prediction of a complicated AHA-LT6 plaque by MR imaging. This study reinforces that complications occur in the soft plaque component of the atherosclerotic plaque, and it can be assumed that the probability of finding a complication increases with the size of the soft plaque thickness.

A previous CTA/MR imaging comparison study showed that the attenuation of plaques with IPH is larger than the attenuation of plaques without IPH (47 versus $43 \mathrm{HU}, P=.02$ ). ${ }^{26}$ However, this study found a large overlap of $\mathrm{HU}$ of plaques with and without IPH, which suggests that HU measurements are not suited to identify complicated plaques. These findings are supported by our study, which did not show any significant differences in HU of complicated and noncomplicated plaques. Initially we had hypothesized that with the use of the minimal HU value obtained from the ROIs, we would be able to better differentiate hemorrhagic from lipid-rich necrotic core lesions; however, that was not supported by our data.

Interestingly, when all the lesion types containing a lipid-rich necrotic core were combined, those lesions had a significantly lower attenuation than lesions with predominantly fibrous tissue, such as lesion type III and the soft component of lesion type VII $(P=.02)$. This is consistent with a previous study by de Weert et $\mathrm{al}^{23}$ which showed that CT is able to differentiate lipid from fibrous tissue if lesions are not calcified. Although our study showed that CTA is able to differentiate between lipid and fibrous tissue in noncomplicated plaques, our findings also indicated that CTA is unable to differentiate between lipid-rich lesions with and without complications, thus limiting its clinical use to identify the vulnerable plaque.

Interreader agreement for the maximum soft plaque component thickness and maximum total vessel wall thickness was excellent, which indicates that these measurements are highly repro- ducible. Although orthogonal vessel wall measurements are generally considered more accurate than axial measurements because they are not biased by vessel tortuosity, our results indicate excellent agreement between measurements in axial and orthogonal planes and no systematic difference. In our opinion, axial thickness measurements are preferable because they are less timeconsuming and can be performed easily without postprocessing on a standard PACs work station. Thus, this measurement can be easily adopted into routine clinical practice.

\section{Limitations}

Our patient population had a relatively large percentage of complicated plaques $(23 \%)$. However, this is expected because all patients had a symptomatic TIA or ischemic stroke of the anterior circulation, and strong associations between AHA-LT6 and ischemic stroke have been reported recently. ${ }^{11}$ Although efforts were made to reduce the time between studies, delay in obtaining the MR imaging may have generated confounding variables. However, on the basis of our experience and the experience of others with serial MR imaging, atherosclerotic changes over time are minimal. ${ }^{10,32}$ We do not believe that this altered our results substantially. Of importance, in our clinic, patients with CTA-proven or sonography-proven significant carotid stenosis of $>70 \%$ undergo carotid intervention within 24 hours and were thus excluded from our study.

\section{CONCLUSIONS}

The maximum soft plaque component thickness as measured by carotid CTA has the best discriminatory power to predict the presence or absence of AHA-LT6. All plaques with a soft tissue component $>5.6 \mathrm{~mm}$ were associated with complicated AHALT6 by MR imaging, whereas none of the plaques with a soft tissue component $<2.2 \mathrm{~mm}$ showed a complicated AHA-LT6. Thus, 2.2-mm soft plaque thickness appears to be the ideal cutoff to exclude the presence of complicated AHA-LT6 plaque, though further studies are needed to confirm the reliability of the above stated thresholds. Of particular importance is to confirm that a larger soft plaque component size increases the probability of atherosclerotic plaque complications and thus the probability of clinical strokes.

Disclosures: Martin Dichgans_UNRELATED: Board Membership: Boehringer Ingelheim, EVER Pharma; Consultancy: Bayer, Boehringer Ingelheim, Heel, Bristol-MyersSquibb; Grants/Grants Pending: EU FP7,* DFG,* DLR,* Fondation Leducq,* Vasc.Dem.Res.Found., ${ }^{\star}$ Jackstaedt, ${ }^{\star}$ Corona*; Payment for Manuscript Preparation: Georg Thieme, UpToDate, W. Kohlhammer. Tobias Saam-UNRELATED: Grants/

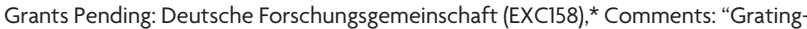
Based Phase Contrast Applied to Atherosclerotic Plaque Imaging using Compact X-Ray Sources"; Payment for Lectures (including service on speakers bureaus): MSD Sharp, DOHME Gmbh, GlaxoSmithKline (*money paid to institution). 


\section{REFERENCES}

1. Redgrave JN, Lovett JK, Gallagher PJ, et al. Histological assessment of 526 symptomatic carotid plaques in relation to the nature and timing of ischemic symptoms: the Oxford plaque study. Circulation 2006;113:2320-28

2. von Sarnowski B, Ludemann J, Volzke H, et al. Common carotid intima-media thickness and Framingham risk score predict incident carotid atherosclerotic plaque formation: longitudinal results from the study of health in Pomerania. Stroke 2010;41:2375-77

3. Freilinger TM, Schindler A, Schmidt C, et al. Prevalence of nonstenosing, complicated atherosclerotic plaques in cryptogenic stroke. JACC Cardiovasc Imaging 2012;5:397-405

4. Randoux B, Marro B, Koskas F, et al. Carotid artery stenosis: prospective comparison of $\mathrm{CT}$, three-dimensional gadolinium-enhanced MR, and conventional angiography. Radiology 2001;220: $179-85$

5. Cai J, Hatsukami TS, Ferguson MS, et al. In vivo quantitative measurement of intact fibrous cap and lipid-rich necrotic core size in atherosclerotic carotid plaque: comparison of high-resolution, contrast-enhanced magnetic resonance imaging and histology. Circulation 2005;112:3437-44

6. Leclerc X, Nicol L, Gauvrit JY, et al. Contrast-enhanced MR angiography of supraaortic vessels: the effect of voxel size on image quality. AJNR Am J Neuroradiol 2000;21:1021-27

7. Ouhlous M, Lethimonnier F, Dippel DW, et al. Evaluation of a dedicated dual phased-array surface coil using a black-blood FSE sequence for high resolution MRI of the carotid vessel wall. J Magn Reson Imaging 2002;15:344-51

8. Aoki S, Nakajima H, Kumagai H, et al. Dynamic contrast-enhanced MR angiography and MR imaging of the carotid artery: high-resolution sequences in different acquisition planes. AJNR Am J Neuroradiol 2000;21:381-85

9. Saam T, Ferguson MS, Yarnykh VL, et al. Quantitative evaluation of carotid plaque composition by in vivo MRI. Arterioscler Thromb Vasc Biol 2005;25:234-39

10. Takaya N, Yuan C, Chu B, et al. Presence of intraplaque hemorrhage stimulates progression of carotid atherosclerotic plaques: a highresolution magnetic resonance imaging study. Circulation 2005; 111:2768-75

11. Parmar JP, Rogers WJ, Mugler JP 3rd, et al. Magnetic resonance imaging of carotid atherosclerotic plaque in clinically suspected acute transient ischemic attack and acute ischemic stroke. Circulation 2010;122:2031-38

12. Alexandrov AV, Vital D, Brodie DS, et al. Grading carotid stenosis with ultrasound: an interlaboratory comparison. Stroke 1997;28: $1208-10$

13. Jahromi AS, Cina CS, Liu Y, et al. Sensitivity and specificity of color duplex ultrasound measurement in the estimation of internal carotid artery stenosis: a systematic review and meta-analysis. $J$ Vasc Surg 2005;41:962-72

14. Saba L, Sanfilippo R, Montisci R, et al. Vulnerable plaque: detection of agreement between multi-detector-row CT angiography and US-ECD. Eur J Radiol 2011;77:509-15

15. Anzidei M, Napoli A, Zaccagna F, et al. Diagnostic accuracy of colour Doppler ultrasonography, CT angiography and blood-pool-enhanced MR angiography in assessing carotid stenosis: a comparative study with DSA in 170 patients. Radiol Med 2012;117:54-71

16. Randomised trial of endarterectomy for recently symptomatic ca- rotid stenosis: final results of the MRC European Carotid Surgery Trial (ECST). Lancet 1998;351:1379-87

17. Barnett HJ, Taylor DW, Eliasziw M, et al. Benefit of carotid endarterectomy in patients with symptomatic moderate or severe stenosis: North American Symptomatic Carotid Endarterectomy Trial Collaborators. N Engl J Med 1998;339:1415-25

18. Halliday A, Mansfield A, Marro J, et al. Prevention of disabling and fatal strokes by successful carotid endarterectomy in patients without recent neurological symptoms: randomised controlled trial. Lancet 2004;363:1491-502

19. de Weert TT, Cretier S, Groen HC, et al. Atherosclerotic plaque surface morphology in the carotid bifurcation assessed with multidetector computed tomography angiography. Stroke 2009;40: $1334-40$

20. Saba L, Caddeo G, Sanfilippo R, et al. CT and ultrasound in the study of ulcerated carotid plaque compared with surgical results: potentialities and advantages of multidetector row CT angiography. AJNR Am J Neuroradiol 2007;28:1061-66

21. Saba L, Caddeo G, Sanfilippo R, et al. Efficacy and sensitivity of axial scans and different reconstruction methods in the study of the ulcerated carotid plaque using multidetector-row CT angiography: comparison with surgical results. AJNR Am J Neuroradiol 2007; 28:716-23

22. Furtado AD, Adraktas DD, Brasic N, et al. The triple rule-out for acute ischemic stroke: imaging the brain, carotid arteries, aorta, and heart. AJNR Am J Neuroradiol 2010;31:1290-96

23. de Weert TT, Ouhlous M, Meijering E, et al. In vivo characterization and quantification of atherosclerotic carotid plaque components with multidetector computed tomography and histopathological correlation. Arterioscler Thromb Vasc Biol 2006;26:2366-72

24. Wintermark M, Jawadi SS, Rapp JH, et al. High-resolution CT imaging of carotid artery atherosclerotic plaques. AJNR Am J Neuroradiol 2008;29:875-82

25. Saba L, Sanfilippo R, Pirisi R, et al. Multidetector-row CT angiography in the study of atherosclerotic carotid arteries. Neuroradiology 2007;49:623-37

26. U-King-Im JM, Fox AJ, Aviv RI, et al. Characterization of carotid plaque hemorrhage: a CT angiography and MR intraplaque hemorrhage study. Stroke 2010;41:1623-29

27. Walker LJ, Ismail A, McMeekin W, et al. Computed tomography angiography for the evaluation of carotid atherosclerotic plaque: correlation with histopathology of endarterectomy specimens. Stroke 2002;33:977-81

28. Saba L, Sanfilippo R, Sannia S, et al. Association between carotid artery plaque volume, composition, and ulceration: a retrospective assessment with MDCT. AJR Am J Roentgenol 2012;199:151-56

29. Cai JM, Hatsukami TS, Ferguson MS, et al. Classification of human carotid atherosclerotic lesions with in vivo multicontrast magnetic resonance imaging. Circulation 2002;106:1368-73

30. Saba L, Sanfilippo R, Pascalis L, et al. Carotid artery wall thickness and ischemic symptoms: evaluation using multi-detector-row CT angiography. Eur Radiol 2008;18:1962-71

31. Magge R, Lau BC, Soares BP, et al. Clinical risk factors and CT imaging features of carotid atherosclerotic plaques as predictors of new incident carotid ischemic stroke: a retrospective cohort study. AJNR Am J Neuroradiol 2013;34:402-09

32. Kwee RM, Truijman MT, van Oostenbrugge RJ, et al. Longitudinal MRI study on the natural history of carotid artery plaques in symptomatic patients. PLoS One 2012;7:e42472 